\title{
Integrating Poison Center Data into Oregon ESSENCE using a Low-Cost Solution
}

\author{
Robert Laing* and Melissa Powell \\ Public Health Division, Oregon Health Authority, Portland, OR, USA
}

\section{Objective}

To enhance Oregon ESSENCE's surveillance capabilities by incorporating data from the Oregon Poison Center using limited resources.

\section{Introduction}

Oregon Public Health Division (OPHD), in collaboration with the Johns Hopkins University Applied Physics Laboratory, implemented Oregon ESSENCE in 2012. Oregon ESSENCE is an automated, electronic syndromic surveillance system that captures emergency department data. To strengthen the capabilities of Oregon ESSENCE, OPHD sought other sources of health-outcome information, including Oregon Poison Center (OPC). In the past, Oregon's surveillance staff manually monitored OPC data on the National Poison Data Service (NPDS) website. Although functional, it was not integrated into Oregon's syndromic surveillance system and required epidemiologists to assess alerts on individual calls. To achieve data integration, OPHD pursued an automated solution to deliver OPC data into Oregon ESSENCE. OPHD's growing interoperability infrastructure fostered development of a low-cost, reliable solution to automate the integration of these data sources.

\section{Methods}

OPC facilitated OPHD's access to the free-of-charge NPDS web service with an approval request and a data use agreement. OPHD uses the Rhapsody Integration Engine 6.2.1 (Orion Health, Auckland, $\mathrm{NZ}$ ) as its primary data transfer and translation mechanism. OPHD leveraged its existing Rhapsody installation to automatically request data from the NPDS web service daily. Each request contains custom search parameters that query calls from the previous day ( 24 hours). The service returns an XML file containing poison center call data with multiple nodes of related data. Rhapsody uses a JavaScript 'filter' to parse each call and its related data. The Oregon ESSENCE backend SQL database contains a parent table for the call and child tables for the related data (Clinical Effects, Routes, Scenarios, Therapies, and Generic Codes). Rhapsody inserts data into each of these backend SQL tables.

\section{Results}

Oregon ESSENCE displays OPC data through its web interface for interpretation by OPHD's syndromic surveillance epidemiologists. Integrating NPDS data into Oregon ESSENCE allows OPHD staff to timely monitor data in an automated, routine manner. Syndromic surveillance staff first assess alerts generated by Oregon ESSENCE. Alerts that require follow-up trigger a call between OPHD epidemiologists and OPC. Oregon is the first state to use the NPDS web service to upload poison center data into Oregon ESSENCE.

\section{Conclusions}

Oregon's successful integration of the NPDS web service data into Oregon ESSENCE is the first known of its kind. It leverages OPHD's growing infrastructure of interoperability software applications and staff expertise to create a cost-effective and sustainable solution that can be easily adapted by other public health agencies.

\section{Keywords}

Interoperability; ESSENCE; Poison Data; Oregon; NPDS

\section{Acknowledgments}

Thank you to Kyle Ryff, MPH; Melissa Powell, MPH; Michelle Barber, MS; and Laurel Boyd, MPH, all of whom spent significant time in the development of OPHD's Rhapsody engine and informatics infrastructure. This publication was supported by Cooperative Agreements, number 1U500E000068-01, funded by the Centers for Disease Control and Prevention. Its contents are solely the responsibility of the authors and do not necessarily represent the official views of the Centers for Disease Control and Prevention or the Department of Health and Human Services.

\author{
${ }^{*}$ Robert Laing \\ E-mail: robert.s.laing@state.or.us
}

